\section{Drops that buckle}

Ludovic Pauchard, Jean-Pierre Hulin and Catherine Allain* Laboratory FAST, Bât. 502, Campus Universitaire 91405 Orsay Cedex, France

*corresponding author: allain@fast.u-psud.fr

$T^{\mathrm{l}}$ he collapse of structures under the effect of their weight or other mechanical stress is of interest in many domains of mechanical and civil engineering [1]. Recently such phenomena have also been approached theoretically in the physics of instabilities dealing with shell and plate geometries [2]. When a structure, subjected to compression, displays large displacements transverse to the load, it is said to buckle [3]. A simple demonstration can be obtained by pressing the opposite edges of a flat sheet towards each other. This instability can be found in various systems, particularly when the volume enclosed within a thin and elastic envelope is made to decrease: collapse of concrete domes, vessels, etc. In general, for small volume variations the shape of the envelope remains the same (the flat sheet is kept plane). However for variations larger than a threshold value, deformations leading to variations of curvature can take place (the flat sheet bends). In this case, the inner volume can become very small with the outer surface displaying large wrinkles.

It has been recently shown that the buckling process is not only relevant to macroscopic systems that can be described through classical mechanics approaches. It is also important in a large variety of systems displaying a broad range of characteristic length scales: for example, amphiphilic or biological polymerized membranes or the buckling-driven delamination of metallic films, or morphology of certain leaves of plants, to name some.

The present work deals with the drying of sessile drops (drops deposited upon a solid surface) of a polymer solution which is controlled by the diffusion of solvent vapour in air. The drying process first leads to the formation of a superficial rigid skin to be discussed below. In such systems, the buckling process results from complex spatial and temporal evolutions leading thus to various unexpected final shapes of the drops. For instance, the diagram in Figure 1 displays the top views of the drop shapes observed at the final stage of the drying process under different conditions (different initial contact angles of the drop onto the substrate and different relative humidity of the surroundings of the sessile drop).

The experiments were carried out using solutions of Dextran, a water-soluble polysaccharide, at high concentration $\omega_{\mathrm{p}}=$ $0.40 \mathrm{~g} / \mathrm{g}$. At room temperature, the pure dry polymer is glassy and mechanically hard (its glass transition temperature is $220 \pm 10^{\circ} \mathrm{C}$ ). For a solution, the glass transition temperature ranges between that of the pure solvent and that of the pure polymer; it increases continuously with the polymer concentration. At a given intermediate temperature, there will be a concentration $\omega_{\mathrm{pg}}$ such that the solution is fluid when $\omega_{\mathrm{p}}<\omega_{\mathrm{pg}}$ and glassy when $\omega_{\mathrm{p}}>\omega_{\mathrm{pg}}$. Also, during the drying process due to solvent removal, the polymer concentration increases with time and the solution, which is initially fluid, becomes glassy. The geometry considered here is that of a drop which is deposited onto a glass slide; the wetting properties of the substrates can be modified in order to vary the initial contact angle, $\theta_{0}$. The setup is placed inside a glove box inside which the relative humidity, $\mathrm{RH}$, is controlled. Following solvent

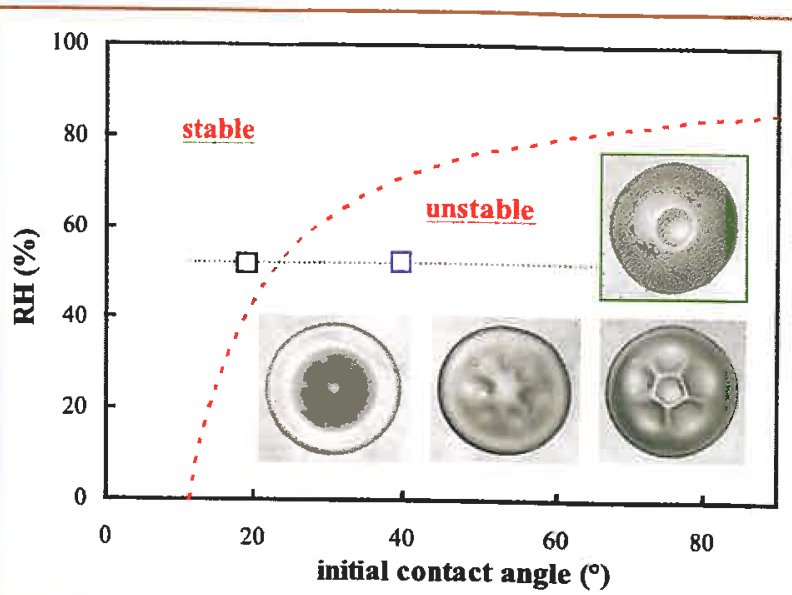

$\triangle$ Fig. 1: Diagram showing the instability occurrence for sessile drops during solvent loss under different conditions of initial contact angle $\left(\theta_{0}\right)$ and humidity rate $(\mathrm{RH})$. The red dashed line (theoretical prediction) limits the "stable" and the "unstable" behaviour where drops get strong distortions during the drying process. Insets: images in top view taken at the final stage of the drying process; their location in the diagram corresponds to the conditions $\left(\theta_{0}, \mathrm{RH}\right)$ necessary to form them. The diameter of the drop contact base is about $5 \mathrm{~mm}$.

removal, polymer deposition and adhesion onto the substrate leads to a strong pinning of the three-phase line $[4,5]$ : the drop evaporates with a constant contact area with the substrate, unlike a pure solvent drop which recedes with a constant contact angle. Inside the drop volume the polymer concentration is not uniform with time but becomes higher near the outer surface where evaporation takes place (Figure 2). So, when the polymer concentration reaches $\omega_{\mathrm{pg}}$ in this region, a glassy skin forms. This skin is too thin to change significantly the evaporation rate (diffusion continues through the thickness of the skin). Then the drop surface is rigid whereas the inner volume decreases due to evaporation: a buckling process can therefore take place. Note that, for the instability to occur, it is necessary that the core of the drop be still fluid when the glassy skin forms at the drop surface. By studying the variations with time of the profiles, volume and surface area of the drops, we have confirmed that the instability occurring in the experiments is indeed a buckling instability. By comparing the characteristic drying time and the time required to form a glassy skin at the drop surface, it is possible to obtain a criterion for the observation of the instability (red dashed line in the diagram of Figure 1) [6].

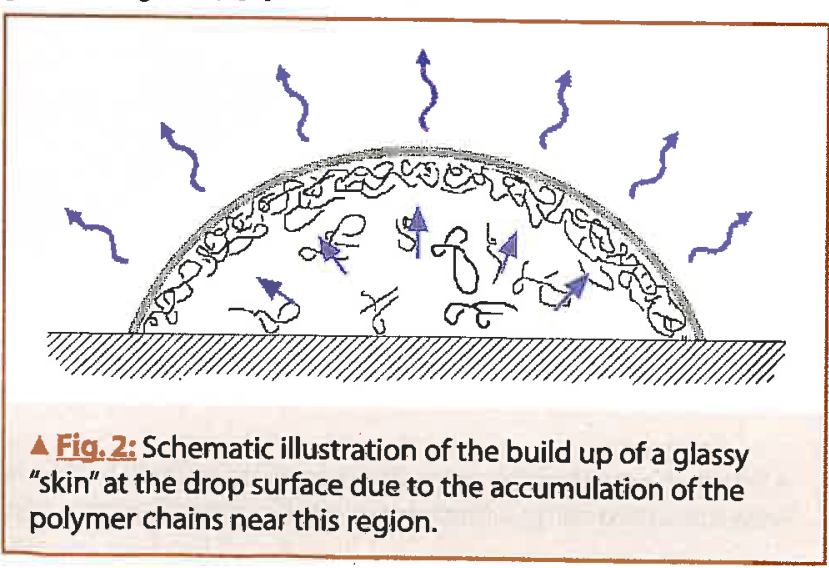




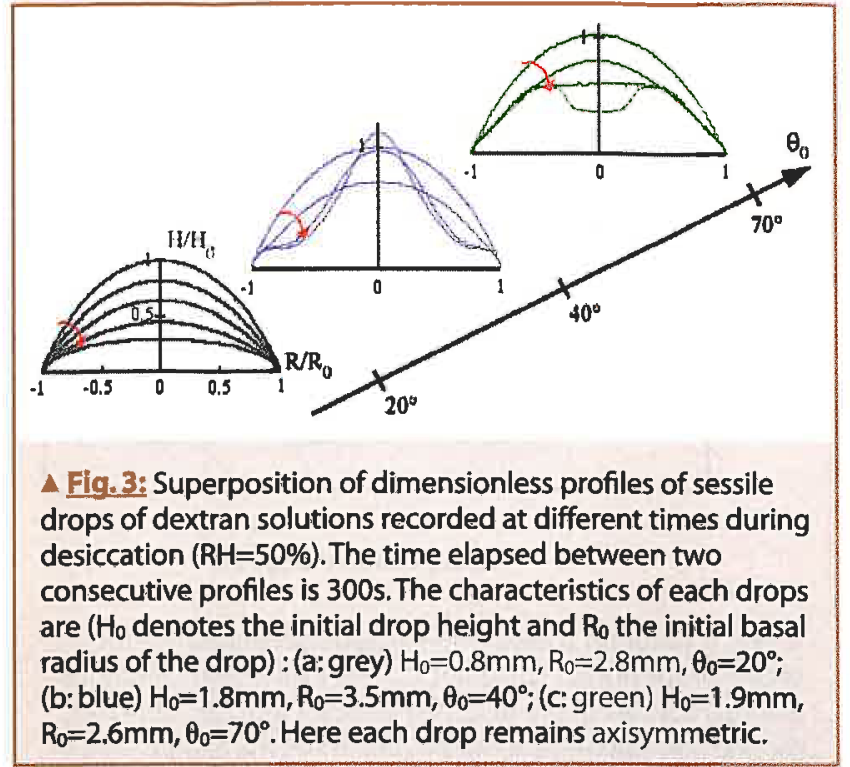

Let us now analyse the distortions of the drop shapes when the initial contact angle is varied at fixed relative humidity (horizontal grey dashed line of Figure $1-\mathrm{RH}=50 \%$ ). For low contact angles $\left(\theta_{0}<20^{\circ}\right)$, the drop progressively flattens following solvent evaporation. The shape of the drop is not strongly modified, as shown by the superposition of profiles recorded at different time on Figure 3a (conditions corresponding to the grey left square in the diagram in Figure 1). The region of the diagram in Figure 1 where this behaviour can be observed is named "stable"; in the other regions large distortions of the sessile drops are observed ("unstable behaviour"). For higher contact angles $\left(20^{\circ}<\theta_{0}<60^{\circ}\right)$, the drop flattens in a stage and the height of the apex decreases at first but starts to increase again after a certain time (see superposition of profiles in Figure $3 \mathrm{~b}$ corresponding to the blue right square in the diagram in Figure 1). For still higher contact angles $\left(\theta_{0}>60^{\circ}\right)$, the drop flattens as the apex height progressively decreases following the same trend as in the previous cases. Then the top of the drop decreases at a faster rate and a depression forms leading to an inversion of the curvature (Figure $3 c$ and the right top inset in Figure 1 surrounded with a green line) [6]. Such a curvature inversion has also been observed in the mechanics of a "ping-pong" ball i.e. in the compression of an elastic spherical shell [7]. At the final stage of the drying the drop shape displays a circular fold, with a dip at the centre of the droplet, only observable on profilometry measurements (Figure 4a) [8].

Let us now consider again the influence of the initial contact angle on the distorted shape of the drop but this time at a lower relative humidity $(\mathrm{RH}=30 \%)$. This time, the rate of evaporation is higher. As a consequence, the polymers rapidly accumulate at the drop surface (the concentration gradient having less time to relax) and the glassy skin forms earlier in the course of the drying than at a higher relative humidity [6]. Thus the buckling process takes place for a smaller contact angle (see Figure 1). Moreover, for initial contact angle such as $20^{\circ}<\theta_{0}$, the large volume variation that will take place after skin formation results in secondary instabilities such as wrinkling, breaking the axisymmetry of the distorted drops. For $20^{\circ}<\theta_{0}<60^{\circ}$, at first the drop flattens and the height of the drop decreases as shown in dimensionless coordinates in Figure 5. Then the apex height quickly increases and reaches a maximum value (peak formation). After a few minutes secondary instabilities take place and break the axisymmetry of the drop: radial wrinkles appear all around the peak (see Figure $4 \mathrm{~b}$ and inset in Figures 1 and 5). For higher initial contact angle $\left(\theta_{0}>60^{\circ}\right)$, the curvature at the top of the drop changes sign during the drying process as already observed for $\mathrm{RH}=50 \%$. Then a

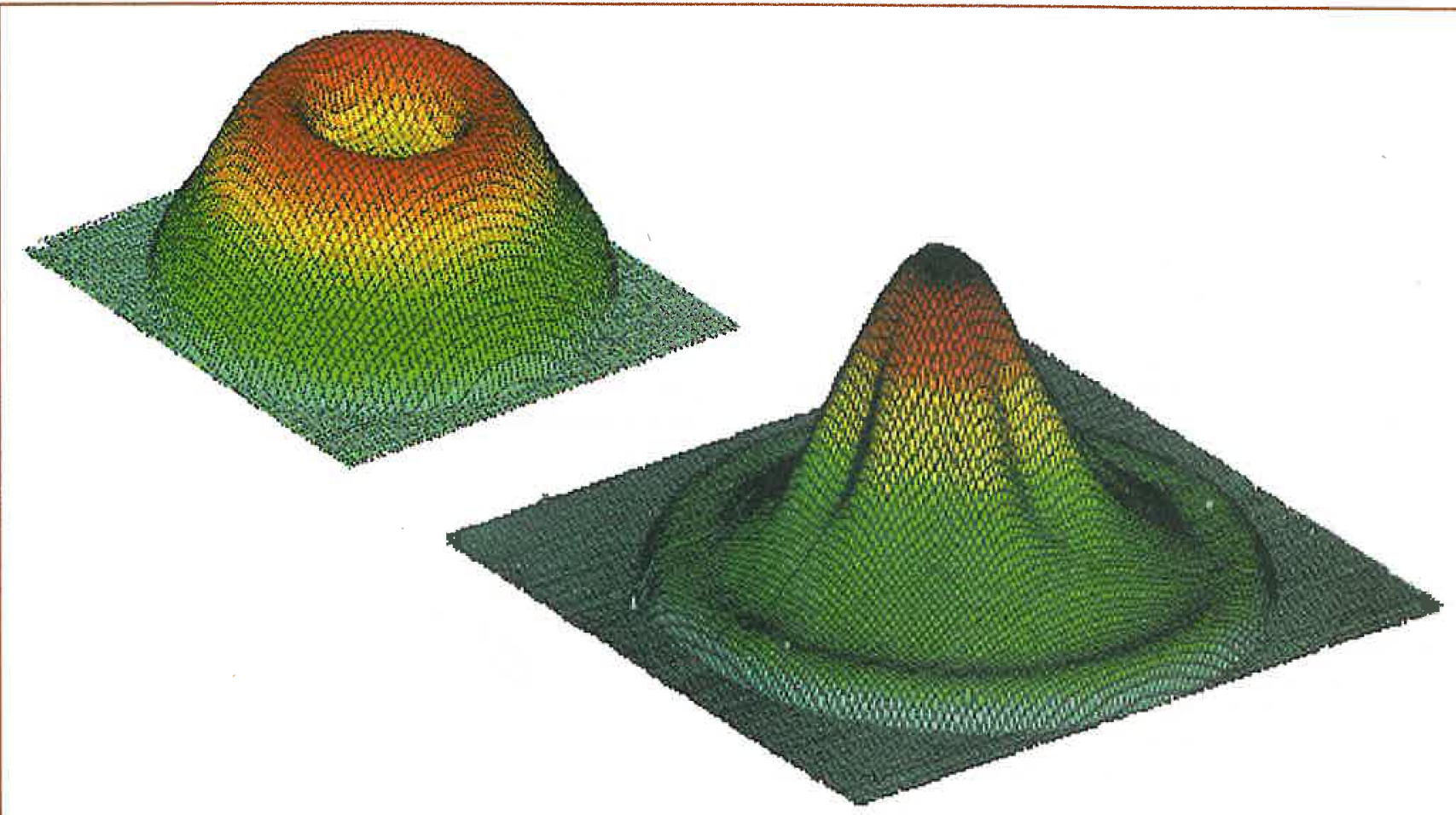

$\triangle F i g .4: 30$ maps of the drop corresponding to (a) $R H=50 \% ; \theta_{0}=70^{\circ} ; R 0=2.6 \mathrm{~mm}$ and (b) $\mathrm{RH}=30 \% ; \theta_{0}=40^{\circ} ; \mathrm{R} 0=2.9 \mathrm{~mm}$. These profiles were measured using a mechanical profilometer (length scales are amplified in the $z$ direction to improve legibility). 